OPEN ACCESS

Edited by:

Reza Lashgari,

Shahid Beheshti University, Iran

Reviewed by:

Beatrice Ludovica Ritondo,

University of Rome Tor Vergata, Italy

Gigi Lam,

Hong Kong Shue Yan University,

Hong Kong SAR, China

*Correspondence:

Varut Vardhanabhuti varv@hku.hk

Specialty section:

This article was submitted to

Pulmonary Medicine,

a section of the journal

Frontiers in Medicine

Received: 03 May 2021

Accepted: 19 October 2021

Published: 17 November 2021

Citation:

Ng KS and Vardhanabhuti V (2021) Chest-Related Imaging Investigations During Multiple Waves of COVID-19

Infection in Hong Kong.

Front. Med. 8:704515.

doi: 10.3389/fmed.2021.704515

\title{
Chest-Related Imaging Investigations During Multiple Waves of COVID-19 Infection in Hong Kong
}

Kei Shing $\mathrm{Ng}$ and Varut Vardhanabhuti*

Department of Diagnostic Radiology, Li Ka Shing Faculty of Medicine, The University of Hong Kong, Hong Kong, Hong Kong SAR, China

Background: The COVID-19 pandemic has caused significant disruption to healthcare worldwide. In this study, we aim to quantify its impact of chest related radiological procedures over the different waves of local infection in Hong Kong across the territory's public hospitals.

Methods: This was an observational study enrolling patients between January 2017 and December 2020. Consecutive population-based chest radiographs, CT, US, and interventional radiology $(\mathrm{IR})$ procedures were obtained public hospitals across Hong Kong.

Results: A significant reduction of 10.0\% $(p<0.001)$ in the total number of chest radiographs was observed. Non-significant reduction of 2.5\% ( $p=0.0989), 39.1 \%$ ( $p=0.2135)$, and $1.9 \%(p=0.8446)$ was observed for Chest CT, Chest US, and Chest IR procedures, respectively, in 2020 compared to the projected values.

Conclusion: Although, it was anticipated that there would be a significant impact to health services caused by the pandemic, for chest-related investigations in Hong Kong, the impact was not as severe. Quantitative analysis could help with future planning and public health decision making.

Keywords: COVID-19, X-ray, CT, chest ultrasound, interventional radiology

\section{INTRODUCTION}

Significant impact of coronavirus disease 2019 (COVID-19) was observed in the year 2020. In particular, medical services have been reduced or have been redirected toward caring for patients with coronavirus disease 2019 (COVID-19) (1). The first local COVID-19 case in Hong Kong was reported on January 23, 2020. On October 18, 2021, 12,294 confirmed cases (2,952 imported cases and 9,342 local cases) and 213 deaths were reported in the territory. In terms of medical imaging, varying impacts on chest ultrasound (2) and interventional radiology (3-6) were reported globally, but few studies have examined the impact at a population level over a prolonged period. The effects 
TABLE 1 | The actual, predicted number using the time series method and percentage change of chest-related procedures in 2020.

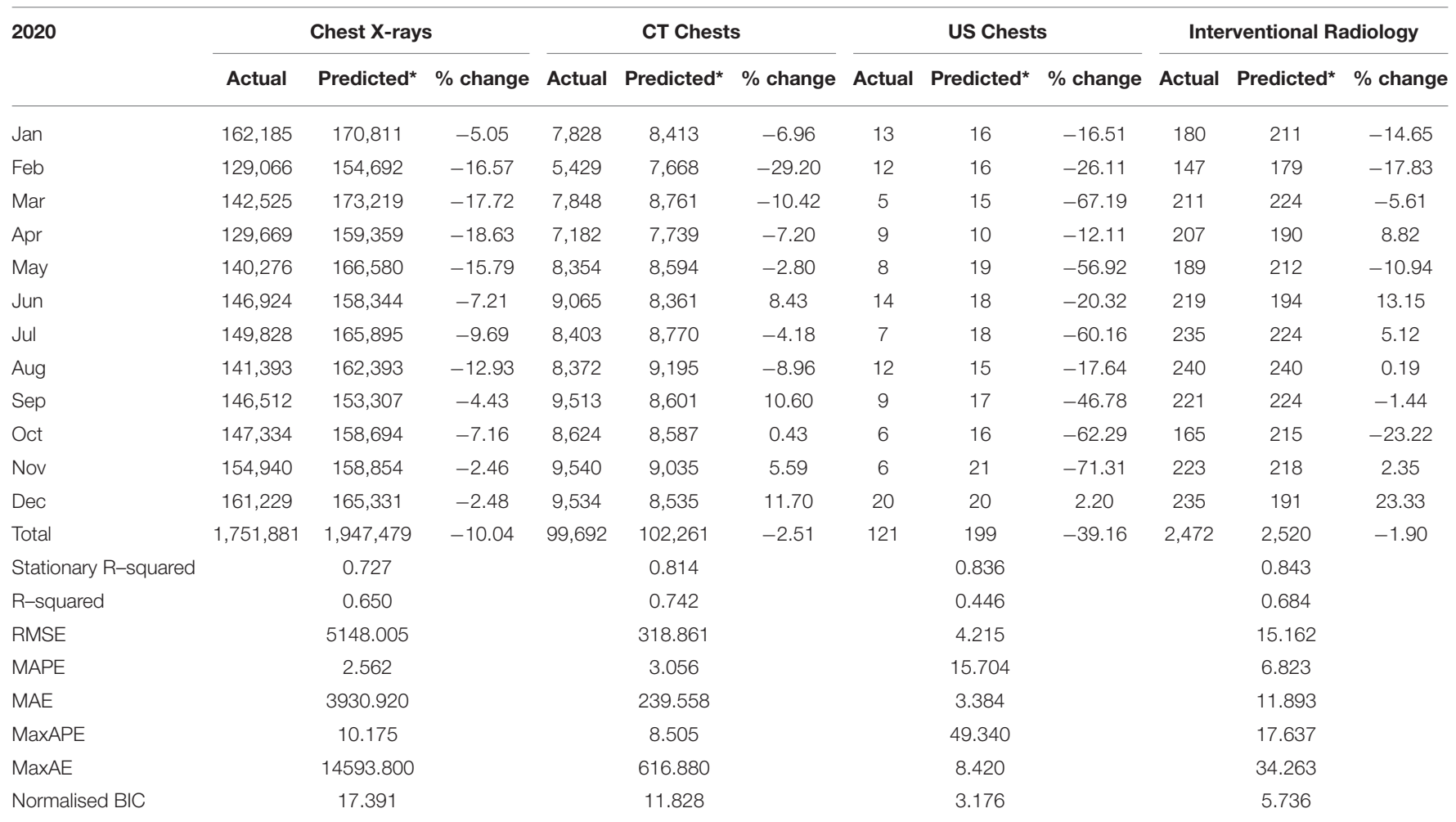

RMSE, Root mean square error; MAE, Mean absolute error; MaxAPE, Maximum absolute percentage error; MaxAE, Maximum absolute error; Normalised BIC, Normalised Bayesian information criterion.

"The predicted values were rounded to nearest integer, and the percentage change was based on the full digits of the predicted value.

of chest X-ray and chest CT have also not been quantitatively reported at a population scale. In this study, we systematically examined territory-wide chest-related imaging investigational data in the Hong Kong public hospitals database and compare the impact in 2020 with the prior 3 years.

\section{METHODS}

The Hong Kong Hospital Authority Clinical Data Analysis and Reporting System (CDARS) was used to conduct a retrospective search of electronic records of patients using a national database. The study was approved by the institutional review board, and, due to the retrospective nature of the study, the patient consent was not required. The study retrieved data between January and December of the years 2017 and 2020, covering chest imaging investigations, specifically chest radiographs, chest computed tomography (CT), chest ultrasound (US), and chest interventional radiology (IR) procedures. The time series model was conducted on the data from the prior years of 2017-2019 to estimate the predicted number of procedures in 2020 (Time Series Modeller, SPSS, IBM Corp., Armonk, N.Y., USA). Differences in counts between the predicted and actual values were compared using a generalised linear model with the Poisson distribution. Statistical significance was set as $p<0.05$. The analysis was performed using R Studio version 1.3 (rstudio.com, Boston, Massachusetts, United States).

\section{RESULTS}

In 2020 , there were a total number of $1,751,881$ chest radiographs, 99,692 chest CT, 121 chest US, and 2,472 chest IR procedures performed from $35,19,11$, and 18 hospitals, respectively, across public hospitals in Hong Kong. A significant reduction of 10.\% $(p<0.001)$ in the total number of chest radiographs was observed in 2020 compared to the predicted value based on the time series model. Non-significant reduction of $2.5 \%$ ( $p=0.0989), 39.1 \%$ $(p=0.2135)$, and $1.9 \%(p=0.8446)$ was observed for Chest CT, Chest US, and Chest IR procedures in 2020 compared to the predicted values based on the time series model (see Table 1, Figure 1).

Monthly analysis revealed that there was an overall trend of a marked initial reduction in the early months at the start of pandemic. Cases were returned to almost a prepandemic level during the easing of social restrictions but reduced again in July 2020, coinciding with the reemergence of infection.

For chest radiograph, reduction was observed in all the months of 2020 compared to predicted numbers (with 


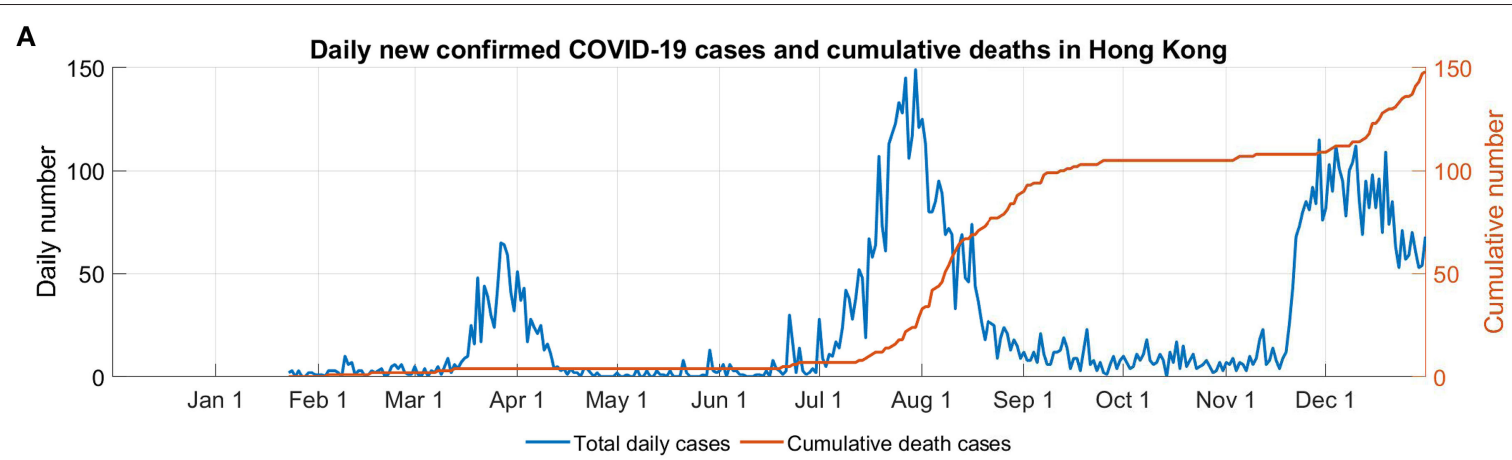

B

The monthly percentage change of chest related procedures in 2020

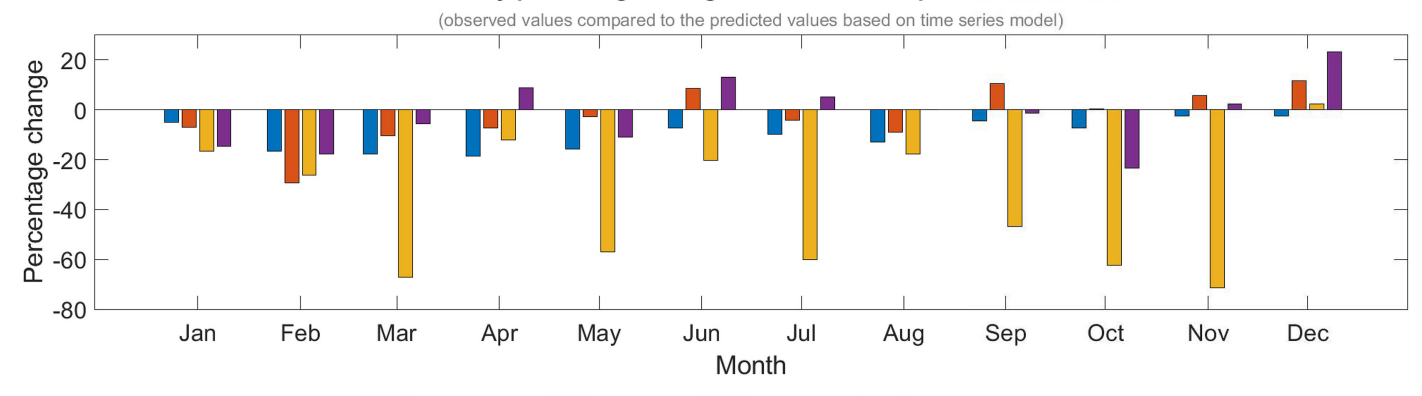

C

Chest X-rays $\square$ CT Chests $\square$ US Chests $\square$ Interventional Radiology

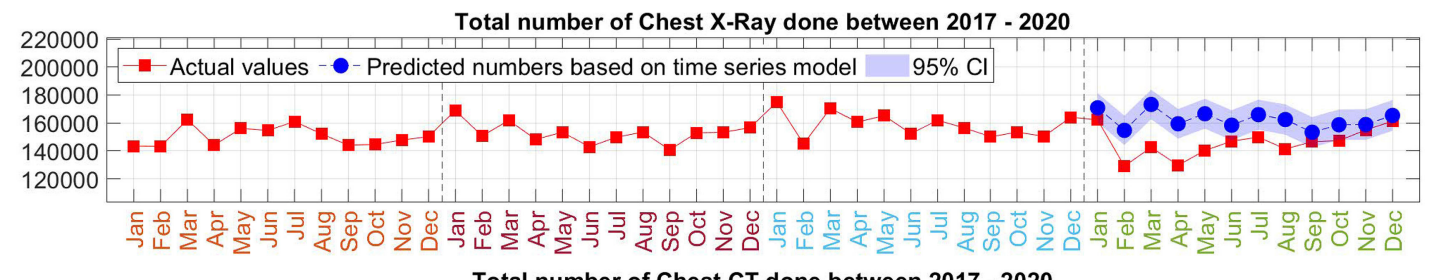

Total number of Chest CT done between $2017-2020$

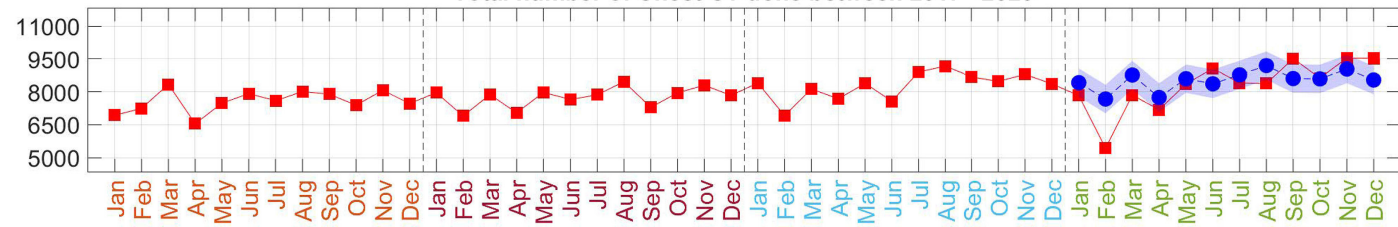

Total number of Chest US done between $2017-2020$

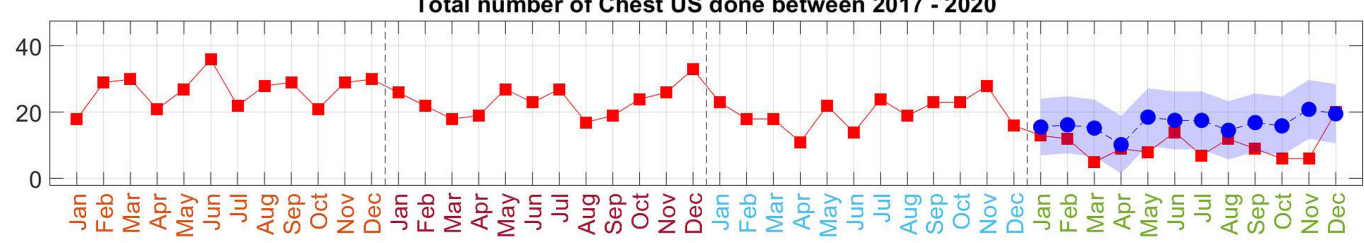

Total number of Interventional Radiology between 2017 - 2020

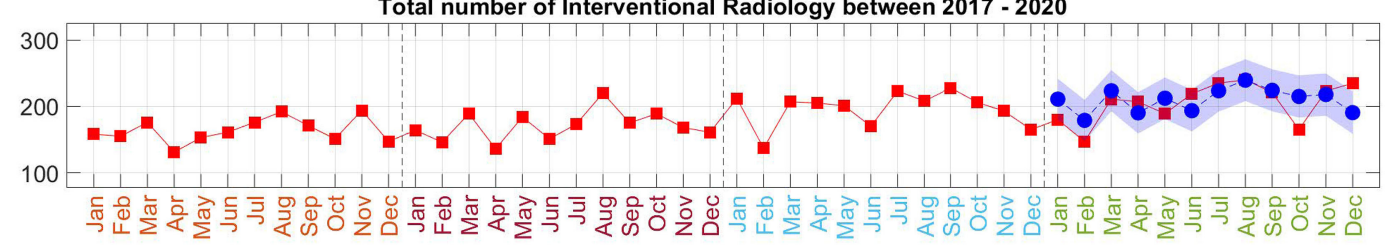

FIGURE 1 | (A) Daily newly confirmed COVID-19 cases and cumulative deaths in Hong Kong, (B) The monthly percentage change of chest-related procedures in 2020, (C) Time series analysis of the chest-related procedures in 2020. 

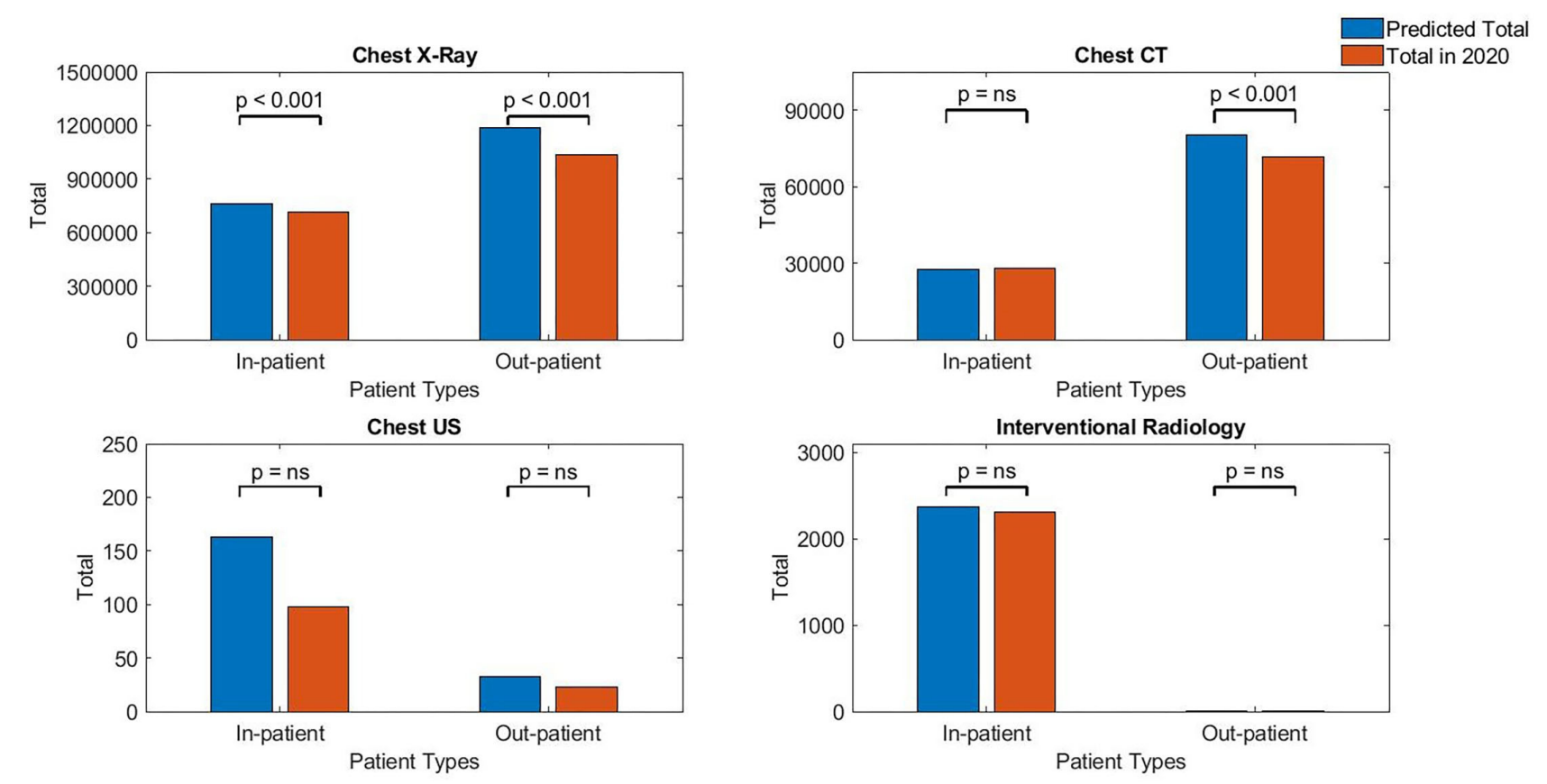

FIGURE 2 | Chest-related investigations for different patient types in 2020.

negative $\%$ in all the months). For chest CT, although marked reduction was observed initially, there was an increase in number of investigations in the months of June, and September to December, and, by the year end, there was only minimal overall reduction of $2.5 \%$ for 2020 . For chest US, the monthly changes showed a double-digit percentage reduction in January to November $(-12.1$ to $-71.3 \%)$ in 2020 with only a mild catchup (2.2\%) in December. For chest IR, monthly analysis showed a reduction in the period of January to March in 2020, and subsequently increased in the following months with another reduction in October, with increasing numbers again toward year end. As a result, the overall yearly reduction was mild $(-1.9 \%, p=0.8446)$.

When analysis was done based on patient types, for chest radiographs, the impact was more for outpatient investigations $(-12.45 \%, p<0.001)$ compared with inpatient $(-6.40 \%$, $p<0.001)$. For Chest CT, outpatient investigations were more affected with overall reduction observed $(-11.05 \%, p<$ $0.001)$ compared to mild increase for inpatient investigations $(+2.7 \%, p=0.3716)$. For Chest US, the inpatient contributed a marked reduction $(-39.8 \%, p=0.2516)$ compared to a modest reduction for outpatient $(-28.7 \%, p=0.7210)$. Finally, for chest IR, the reduction by type of a patient was mild for inpatient $(-2.7 \%, p=0.7882)$ and large for outpatient $(-50.7 \%, p=0.7720)$. However, the total chest IR done for outpatient was $<10$ procedures, which may be regarded negligibly small to draw firm conclusion (see Figure 2, Table 2).

\section{DISCUSSION}

It was perceived that many radiology departments may observe up to a $50-70 \%$ reduction in imaging volume that may last for several months during the COVID-19 pandemic (7). In our study, during the initial waves, a more pronounced reduction was observed, which was most likely attributed to a tighter initial lockdown and cancellation of procedures, likely due to a more conservative stance taken due to a relative lack of knowledge about the new disease. The conservative stance may have stemmed from a previous encounter of Hong Kong with the SARS outbreak in 2003 (8), when the mortality rate in Hong Kong was $17.04 \%$, which was about three times that of China (4.87\%). Original findings of a high infection incidence and a mortality rate $(2.97 \%)$ among healthcare staff in hospitals (9), as well as a lack of protective gear for hospitals and clinics (10), may have contributed to the extreme vigilance of the government and refusal of patients to attend hospital appointments (11). Anecdotally, this was often found at the local level, where some patients would phone to postpone or cancel appointments.

Despite of the confirmed case numbers being much higher for the subsequent waves of infection in December 2020, the opposite trend was observed with only a minor decline in Chest X-ray and a small increase in CT Chest, US Chest, and IR. This was more likely due to improved preparedness and increased precautions implemented in hospitals as well as more experience in coping with the COVID-19 pandemic in the months following the outbreak. During these times, precautions, such as wearing 
masks, mandatory measurement of body temperature, protective covering for staffs, and frequent hand washing, were in place. The adoption of COVID-19 contact tracing apps for patients and anti-pandemic legislation Cap. 599 on home quarantine and social distancing, and compulsory testing for certain persons introduced by the Hong Kong SAR government were also routine procedures with a high adoption rate in Hong Kong.

There were likely multiple reasons for the reduction in radiological procedures observed. The reason for less impact on chest CT and chest IR procedures may be postulated to be due to the fact that more severely ill patients still required these crucial investigational procedures. In addition, we postulate that there were increasing needs of cross-sectional imaging and interventional procedure requirement for chest-related diseases during the pandemic. There may also have been preferential imaging practises, which caused a reduction of ultrasound procedure, for example, to try to minimise direct patient contact. This may be the reason why there was a reduction in chest US inpatient numbers (although not significantly reduced). It must be noted that the numbers were few, in general, to draw any firm conclusion. It was not possible to clearly identify the reasons (e.g., if this was due to change of practise or behaviour of physicians) due to retrospective nature of the study across multiple sites. Looking ahead, as we move toward living with COVID-19 as endemic infections, continued practises of enhanced precautions may become permanent. Health providers must, therefore, try to be more proactive and anticipate potential impact in the event of an outbreak, and minimise the impact of reduction in radiological investigations as far as possible. There are a few ways that one could do this. First, for urgent investigations, provisions must be put in place to be able to provide these services and minimise the cancellations as far as possible. Second, if cancellations were to occur for non-urgent cases, the healthcare providers should adopt a system that allows for a more rapid rearrangement and, for example, may increase the quota of non-emergency outpatient investigations to offset any reduction of services during the localised outbreaks. Radiologists should work closely with their referral doctors to review and reschedule such examinations.

In conclusion, despite relatively low case numbers in Hong Kong compared to other countries, a significant reduction in chest radiographs investigation was seen, but not for chest CT, chest US, or chest IR procedures when analysed at a population level based on yearly analysis.

\section{DATA AVAILABILITY STATEMENT}

The raw data supporting the conclusions of this article will be made available by the authors, without undue reservation.

\section{ETHICS STATEMENT}

The studies involving human participants were reviewed and approved by Cluster Research Ethics Committee/Institutional Review Board (REC/IRB). Written informed consent for participation was not required for this study in accordance with the national legislation and the institutional requirements. 


\section{AUTHOR CONTRIBUTIONS}

$\mathrm{KN}$ and VV were involved in study conception, statistical analysis, drafting of the initial version of the manuscript, data

\section{REFERENCES}

1. Vardhanabhuti V, Ng KS. Differential impact of COVID-19 on cancer diagnostic services based on body regions: a public facilitybased study in Hong Kong. Int J Radiat Oncol Biol Phys. (2021) 111:331-6. doi: 10.1016/j.ijrobp.2021.05.010

2. Vetrugno L, Baciarello M, Bignami E, Bonetti A, Saturno F, Orso D, et al. The "pandemic" increase in lung ultrasound use in response to Covid-19: can we complement computed tomography findings? A narrative review. Ultrasound J. (2020) 12:39. doi: 10.1186/s13089-020-00185-4

3. Cahalane AM, Cui J, Sheridan RM, Thabet A, Sutphin PD, Palmer WE, et al. Changes in interventional radiology practice in a tertiary academic center in the United States during the coronavirus disease 2019 (COVID-19) pandemic. J Am Coll Radiol. (2020) 17:873-7. doi: 10.1016/j.jacr.2020.05.005

4. Hashmi A, Parikh K, Al-Natour M, Azar N, Sutter C, Ramaiya N, et al. Interventional radiology procedural volume changes during COVID-19 initial phase: a tertiary level Midwest health system experience. Clin Imaging. (2021) 72:31-6. doi: 10.1016/j.clinimag.2020.10.039

5. How GY, Pua U. Trends of interventional radiology procedures during the COVID-19 pandemic: the first 27 weeks in the eye of the storm. Insights Imaging. (2020) 11:131. doi: 10.1186/s13244-020-00938-8

6. Zhong J, Datta A, Gordon T, Adams S, Guo T, Abdelaziz M, et al. The impact of COVID-19 on interventional radiology services in the UK. CardioVasc Intervent Radiol. (2021) 44:134-40. doi: 10.1007/s00270-020-02692-2

7. Cavallo JJ, Forman HP. The economic impact of the COVID-19 pandemic on radiology practices. Radiology. (2020) 296:E1414. doi: 10.1148/radiol.2020201495

8. Leung GM, Ho LM, Lam TH, Hedley AJ. Epidemiology of SARS in the 2003. Hong Kong epidemic. Hong Kong Med J. (2009) 15(Suppl. 9):12-6. collection, and statistical analysis. VV supervised the work and offered significant intellectual contribution. All the authors offered significant intellectual contribution for the last version of the manuscript and approved the final form.

9. Razzak JA, Bhatti JA, Tahir MR, Pasha-Razzak O. Initial estimates of COVID-19 infections in hospital workers in the United States during the first wave of pandemic.(Research Article). PLoS One. (2020) 15:e0242589. doi: 10.1371/journal.pone.0242589

10. Wu H, Huang J, Zhang CJP, He Z, Ming W-K. Facemask shortage and the coronavirus disease (COVID-19) outbreak: Reflection on public health measures. medRxiv. (2020). doi: 10.1101/2020.02.11.20020735

11. LoGiudice SH, Liebhaber A, Schöder H. Overcoming the COVID19 crisis and planning for the future. J Nucl Med. (2020) 61:1096101. doi: $10.2967 /$ jnumed.120.250522

Conflict of Interest: The authors declare that the research was conducted in the absence of any commercial or financial relationships that could be construed as a potential conflict of interest.

Publisher's Note: All claims expressed in this article are solely those of the authors and do not necessarily represent those of their affiliated organizations, or those of the publisher, the editors and the reviewers. Any product that may be evaluated in this article, or claim that may be made by its manufacturer, is not guaranteed or endorsed by the publisher.

Copyright (C) $2021 \mathrm{Ng}$ and Vardhanabhuti. This is an open-access article distributed under the terms of the Creative Commons Attribution License (CC BY). The use, distribution or reproduction in other forums is permitted, provided the original author(s) and the copyright owner(s) are credited and that the original publication in this journal is cited, in accordance with accepted academic practice. No use, distribution or reproduction is permitted which does not comply with these terms. 